\title{
P-wave fracture prediction algorithm using pre-stack data with limited azimuth distribution: A case study in the TZ45 area, Tarim Basin, China
}

\author{
Sam Zandong Sun ${ }^{1 *}$ Wang Zhaoming ${ }^{2}$, Yang Haijun ${ }^{2}$, Xiao Xi', \\ Wang Yueying ${ }^{1}$, Chen Lei ${ }^{1}$ and Yang Pei ${ }^{1}$ \\ ${ }^{1}$ Laboratory for Integration of Geology and Geophysics, China University of Petroleum, Beijing 102249, China \\ ${ }^{2}$ Research Institute of Exploration \& Development, PetroChina Tarim Oilfield Company, Korla, Xingjiang 841000, China \\ (C) China University of Petroleum (Beijing) and Springer-Verlag Berlin Heidelberg 2011
}

\begin{abstract}
Fractured reservoirs always show anisotropic amplitude features, i.e. the reflection amplitude of seismic waves varies with offset and azimuth (AVOZ). A noise attenuation fracture inversion algorithm is presented for fracture detection based on P-wave AVOZ. The conventional inversion method always fails when applied to limited azimuth data because of the existence of noise. In our inversion algorithm, special attention is paid to suppressing the noise during inversion, to overcome the limitation of the conventional inversion method on limited azimuth data. Numerical models are employed to illustrate the effectiveness of the method. The inversion algorithm is then applied to Tazhong 45 area field data which is acquired under limited azimuth distribution. Compared with cores and fullbore formation microimage (FMI), the inverted results (fracture density and orientation) are reasonable, suggesting that the inversion algorithm is feasible for fracture prediction in the Tarim Basin.
\end{abstract}

Key words: Tarim Basin, azimuthal anisotropy, AVOZ inversion, fracture detection

\section{Introduction}

The exploration area has been proven very large in the Tarim Basin in China, about $2,100 \mathrm{~km}^{2}$ in the Tabei area and $7,300 \mathrm{~km}^{2}$ in the Tazhong area. The proven reserves in carbonate reservoirs discovered in the Tazhong area are estimated to be $4.8 \times 10^{9}$ tonnes in the past three years.

The key technical problem is how to predict and characterize this type of reservoirs. The storage spaces are mostly secondary, of which about $99 \%$ porosity is from fractures and dissolution caves or holes (Sun et al, 2011a). How to predict fracture development is a question which confronts us.

It is known that vertically aligned fractures in a reservoir could induce $\mathrm{P}$-wave seismic anisotropy. P-wave contains plenty of fracture information which involves amplitude variation versus azimuth, variation of azimuthal travel time and velocity (Crampin et al, 1980; Ruger 1996; 1998; Grechka et al, 1999; Tsvankin, 2001). A lot of work has been done on fracture detection based on P-wave features. Neidell and Cook (1986) applied P-wave data to predict fractures based on differential stacking velocity. Then amplitude versus offset (AVO) was used to study fractured reservoirs. Ruger (1998) gave the law of P-wave amplitude variation

* Corresponding author. email: szd@cup.edu.cn, samzdsun@yahoo.com Received March 18, 2011 in anisotropic media. Mallick et al (1998), Schoenberg et al (1999) and Gray and Head (2000) used a law of amplitude versus azimuth to study fracture mediums. Shen et al (2002) proposed a method of using attributes such as AVO (amplitude versus offset) and FVO (frequency versus offset) to detect fracture orientation in a carbonate reservoir located in the Maporal Field in the Barinas Basin of southwestern Venezuela. Luo and Evan (2004) presented an amplitudebased multiazimuth approach for mapping fractures. AlMarzoug et al (2006) estimated small azimuthal variations in P-wave velocity (maximum 5\%) and a larger variation in azimuthal AVO at the reservoir (larger than 100\%) in two Saudi Arabian field studies.

A well established technique used in fracture prediction is based on Ruger's equation (Ruger, 1998), which describes the reflection amplitude variation with offset and azimuth (AVOZ). This conventional fracture detection method requires the use of wide azimuth data (Wang et al, 2006; Zelewski et al, 2009), because for azimuthally limited data, the existence of noise will make the solution unstable. However, as Ordovician carbonate reservoirs in the Tarim Oilfield underlie a big desert, poor surface conditions lead to very high seismic acquisition cost, and the pre-stack data is azimuth limited due to cost factors. Thus early fracture detection research always fails. It is a challenge if we make use of the current data with limited azimuth distribution.

The algorithm we introduce in this paper is the $\mathrm{P}$-wave 
fracture prediction algorithm using pre-stack data with limited azimuthal distribution, where a noise attenuation method is employed. Then it is applied to the Tazhong 45 area, where the data is acquired under limited azimuthal distribution. Before the inversion is conducted, the data is processed using the flow which could retain the integrity of the amplitude variation information, where the multiples are excluded, the signal to noise ratio is enhanced and the amplitude preserved migration is employed. The inversion results are proven to be reliable and are in good agreement with the geological data. Moreover, it is feasible to detect effective fractures (higher anisotropy, filled with liquid or gas), which suggests that the algorithm presented in this paper is feasible and effective.

\section{The algorithm of 3D pre-stack inversion}

The Ruger P-wave reflection coefficient equation (Ruger, $1998)$ is

$$
\begin{aligned}
& R(i, \phi)=\frac{1}{2} \frac{\Delta Z}{\bar{Z}}+ \\
& \frac{1}{2}\left\{\begin{array}{l}
\frac{\Delta \alpha}{\bar{\alpha}}-\left(\frac{2 \bar{\beta}}{\bar{\alpha}}\right)^{2} \frac{\Delta G}{\bar{G}}+ \\
\left.\Delta \delta^{V}+2\left(\frac{2 \bar{\beta}}{\bar{\alpha}}\right)^{2} \Delta \gamma\right] \cos ^{2}\left(\phi-\phi_{s}\right)
\end{array}\right\} \sin ^{2} i+ \\
& \frac{1}{2}\left\{\begin{array}{l}
\frac{\Delta \alpha}{\bar{\alpha}}+\Delta \varepsilon^{V} \cos ^{4}\left(\phi-\phi_{s}\right)+ \\
\Delta \delta^{V} \sin ^{2}\left(\phi-\phi_{s}\right) \cos ^{2}\left(\phi-\phi_{s}\right)
\end{array}\right\} \sin ^{2} i \tan ^{2} i+\cdots
\end{aligned}
$$

where $\Delta \varepsilon^{V}, \Delta \delta^{V}$ and $\Delta \gamma$ denote the Thomsen parameters; $\alpha$ and $\beta$ are $\mathrm{P}$-wave velocity and S-wave velocity, respectively; $\phi$ is the acquisition azimuth of the survey line; $\phi_{s}$ is the fracture direction; $i$ denotes the incidence angle; $Z=\rho \alpha$ is the vertical P-wave impedance; and $G=\rho \beta^{2}$ denotes the vertical shear modulus.

When the incidence angle is not large, $\sin ^{2} i \tan ^{2} i$ is relatively small, the third term in Eq. (1) can be neglected. Then Eq. (1) can be given by:

$$
R(i, \phi)=P+\left\lfloor B^{i s o}+B^{a n i} \cos \left(\phi-\phi_{s}\right)\right\rfloor \sin ^{2} i
$$

with

$$
\begin{aligned}
& P=\frac{1}{2} \frac{\Delta Z}{\bar{Z}} \\
& B^{i s o}=\frac{1}{2}\left[\frac{\Delta \alpha}{\bar{\alpha}}-\left(\frac{2 \bar{\beta}}{\bar{\alpha}}\right)^{2} \frac{\Delta G}{\bar{G}}\right] \\
& B^{a n i}=\frac{1}{2}\left[\Delta \delta^{V}+2\left(\frac{2 \bar{\beta}}{\bar{\alpha}}\right)^{2} \Delta \gamma\right]
\end{aligned}
$$

where $P$ represents the reflection coefficient of the vertical incident wave; $B^{\text {iso }}$ is the isotropic term in the reflection coefficient gradient which is azimuthally invariant; $B^{a n i}$ represents the anisotropic term in the reflection coefficient gradient and is also the fracture density to be inverted.

A conventional inversion method based on Eq. (2) can be given by further derivation:

$$
\left\{\begin{array}{l}
R(i, \phi)=C_{1}+C_{2} \cos ^{2} \phi \sin ^{2} i+ \\
2 C_{3} \sin \phi \cos \phi \sin ^{2} i+C_{4} \sin ^{2} \phi \sin ^{2} i \\
C_{2}=B^{a n i} \cos ^{2} \phi_{s} \\
C_{3}=B^{a n i} \sin 2 \phi_{s} \\
C_{4}=B^{a n i} \sin ^{2} \phi_{s}
\end{array}\right.
$$

where $C_{1}, C_{2}, C_{3}$ and $C_{4}$ are four unknowns, assuming the fold of the common reflection point (CRP) is $n$, then Eq. (2) can be expressed as:

$$
A X=B
$$

where $A$ denotes the matrix of $n$ rows and 4 columns; $X$ is the column vector which contains $C_{1}, C_{2}, C_{3}$, and $C_{4} ; B$ is the seismic reflection amplitude (without noise). Then the conventional fracture inversion method is to calculate the unknown $X$. Wide azimuth data are needed in order to overcome the noise interference as the existence of noise makes the solution unstable.

In order to make use of the limited azimuth data to conduct the inversion, a special algorithm is needed to suppress the noise interference. We introduce the noise part $N$ to the conventional equation (Eq. (4)), then Eq. (4) is transformed to:

$$
A X=B+N
$$

Through the singular value decomposition (SVD) method, the matrix $A$ in Eq. (5) can be expressed as:

$$
A=U W V
$$

where $U$ is the orthogonal matrix of $n$ rows and 4 columns; $W$ is the diagonal matrix with 4 rows and 4 columns, and of which the diagonal elements are positive or $0 ; V$ is the orthogonal matrix with 4 rows and 4 columns. Calculating the generalized inverse by the SVD method, we obtain:

$$
\begin{aligned}
X & =\left(A^{\mathrm{T}} A\right)^{-1} A^{\mathrm{T}}(B+N) \\
& =V^{\mathrm{T}} W^{-1} U^{\mathrm{T}} B+V^{\mathrm{T}} W^{-1} U^{\mathrm{T}} N
\end{aligned}
$$

By using Eq. (7), the effective signal can be separated from noise. Concretely, it is to multiply $N$ with a small number or to divide it by a large number, then the noise part will approach 0 . The influence of noise on the inversion result can be limited, which enables us to use the limited azimuth data to perform the inversion (Sun et al, 2011b).

\section{The feasibility of the inversion method}

Fig. 1(a) represents a wide azimuth acquisition system 
(top) and a limited azimuth acquisition system (bottom). On the top part of Fig. 1(a), the spots and receivers distribute in the range of 360 degree azimuth, while at the bottom part of Fig. 1(a), spots and receivers cover a limited part of the whole azimuth range.

We divide Fig. 1(b) into three parts: the top part, the middle part and the bottom part. The colored diagrams represent inversion results of the fracture density ( $B^{\text {ani }}$ in Eq. (2), red highest, green lowest), while the black and white diagrams represent the seismogram CMP gathers with 'full azimuth' or 'limited azimuth' marked at the bottom. As it is shown on the top part of Fig. 1(b), for data without noise, the inversion results of the full azimuth data and the limited azimuth data are the same, and both of them show the correct results (weak, strong, weak, strong feature from left to right).
Further, based on the conventional method, for the data with a certain extent of noise, full azimuth data could be used to calculate anisotropy, as is shown in the middle of Fig. 1(b). However, from the bottom of Fig. 1(b) we can conclude that anisotropy could not be inverted properly from the limited azimuth data based on the conventional method (inside the red ellipse there is no anisotropy). However, if we introduce the noise suppression method into the inversion process, the fracture density which is relatively uniform from left to right can be shown (the right map). Therefore, by the noise attenuation method discussed, we can calculate anisotropy on the basis of the limited azimuth distribution data, which could help us to make use of existing limited azimuth data and possibly save some costs in some acquisition layout (Sun et al, 2011b).

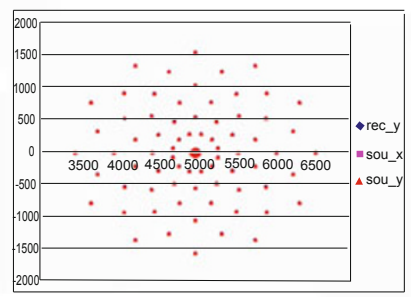

Full azimuth acquisition

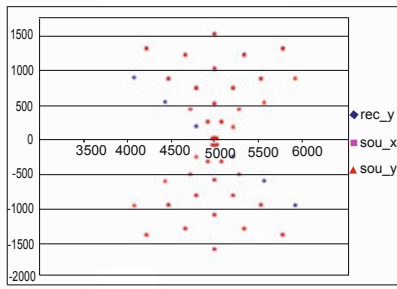

Limited azimuth acquisition

(a)

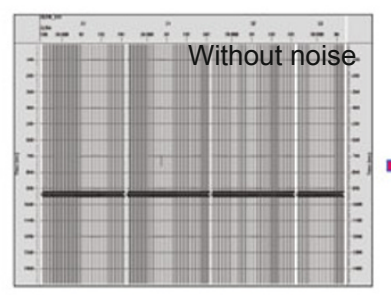

Full azimuth

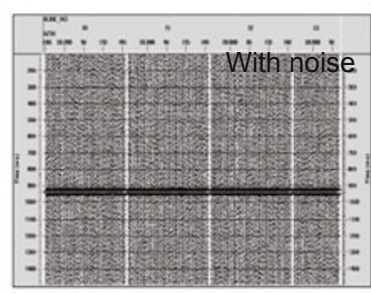

Full azimuth

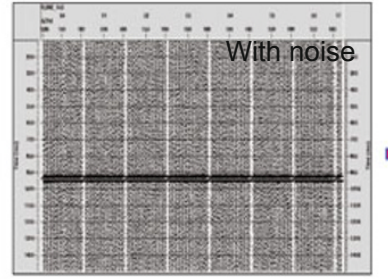

Limited azimuth

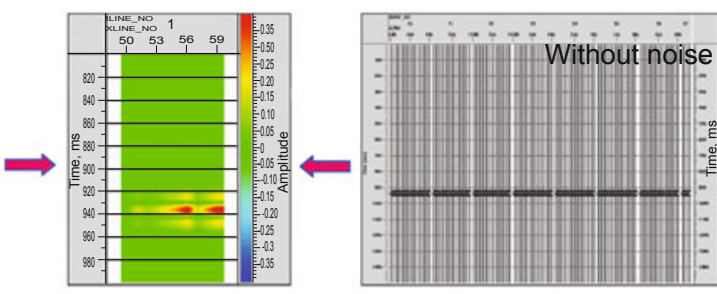

Limited azimuth
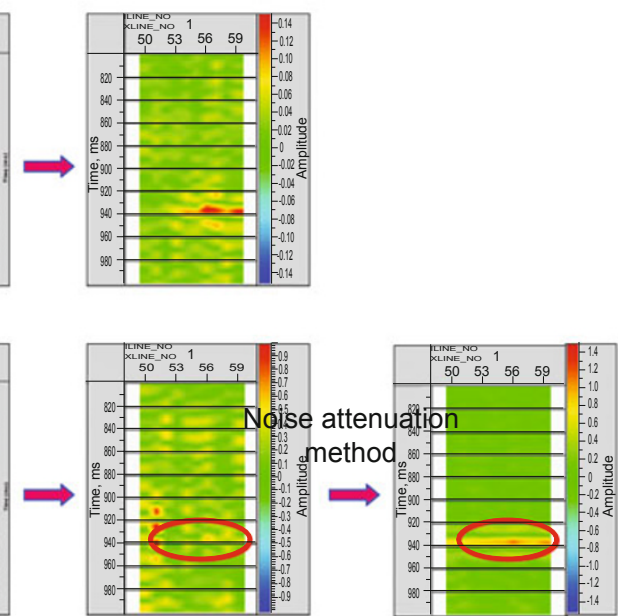

(b)

Fig. 1 Numerical examples illustrating difference between wide azimuth and limited azimuth on azimuthal AVO inversion, showing the effectiveness of the new method in noise suppression

\section{Data processing before inversion}

\subsection{Geometry of 3D field data}

Data quality has been improved as the 3D acquisition system is optimized year by year ever since 2002 in the Tazhong area. Two designing methods are frequently used, those are, 10 lines, 30 shots and 216 traces (every 5 lines), and 12 lines, 36 shots and 216 traces (every 6 lines). Now we analyze the azimuth distribution of acquisition lines and the fold of different offsets, and the analytical results are shown in Fig. 2. From Fig. 2, some conclusions are drawn. Firstly, both of the two acquisition systems have wide ranges of offsets, but the fold of second acquisition system is higher than that of the first one, and both of the two have unequal fold for different offsets. Secondly, the second acquisition system has a relatively larger and more uniform range of azimuth than the first one, but the azimuth range for both of them is mainly centralized in the lateral direction, for the vertical direction, the offsets are smaller, and the fold is lower, the azimuth distribution is not uniform and the range of the fold is limited (Sun and Wang, 2008). 

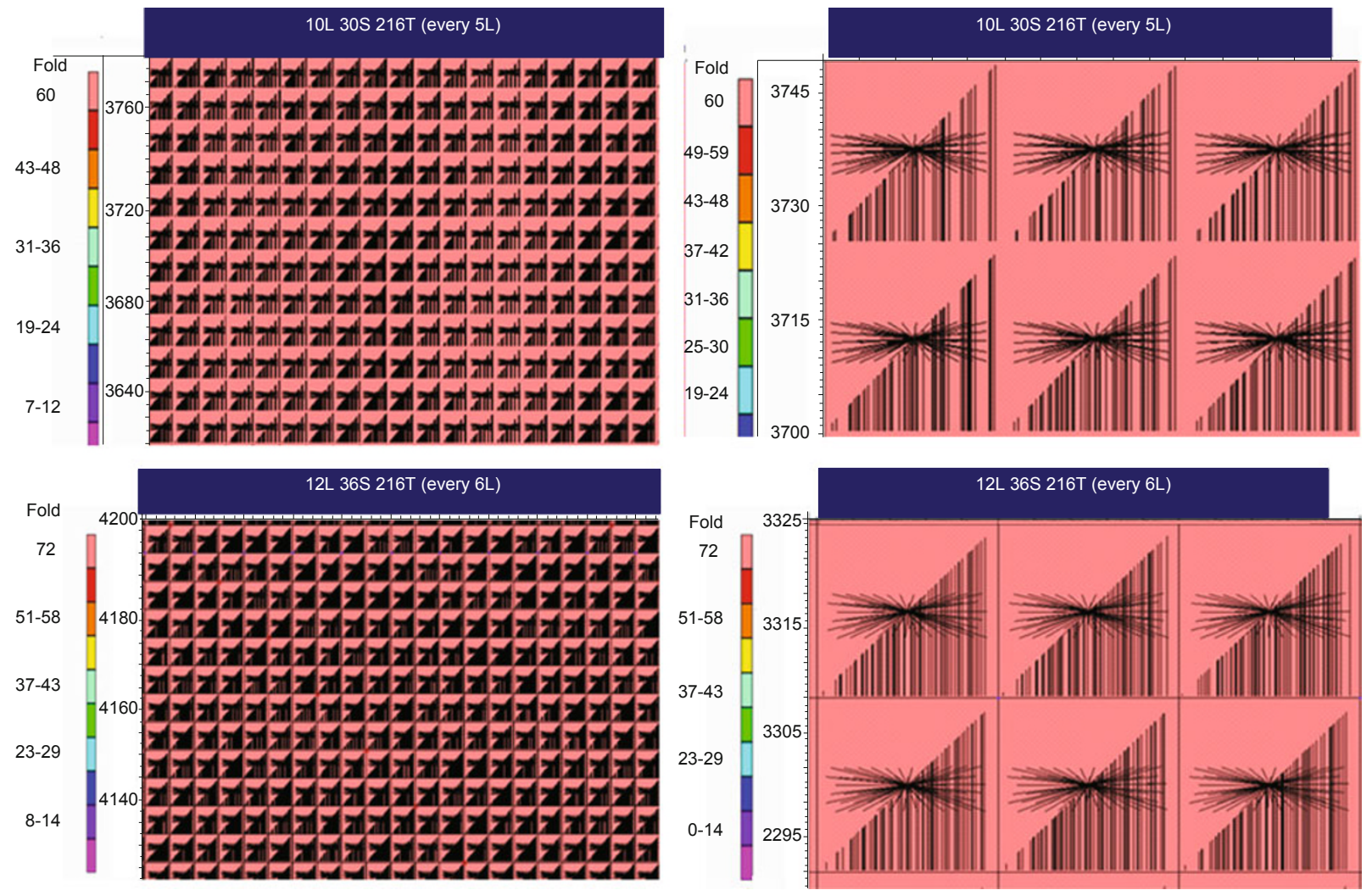

Fig. 2 Analysis of fold and azimuth of CRP gathers in the Tazhong 3D seismic acquisition system (L is short for line, S-shot, T-trace)

\subsection{Problems in the 3D seismic data}

The Tazhong area is characterized by loose dunes in surface, large variation of sand layer thickness. Additionally, Ordovician carbonate reservoirs are characterized by high seismic wave velocity and deep burial with the depth ranging from 4,000 to $7,000 \mathrm{~m}$. Besides, fractures, dissolution pores and caves are highly developed, thus this area shows strong heterogeneity and anisotropy.

Due to the complex geological conditions, seismic data have following characteristics. First, folds in the conventional acquisition system are unequal with high folds in the middle offsets and low in the edge. Second, the azimuth distribution is limited, where the coverage range of azimuth is less than 60 degrees. Third, due to the deep burial, low velocity in the surface and various interferences, the data has low sighal/ noise $(\mathrm{S} / \mathrm{N})$. Considering the data features in the Tazhong area, we present processing flow before inversion, including some key processing methods.

\subsection{Processing flow of the 3D data}

Considering the complexity of seismic data in the Tazhong area, data processing is especially important before anisotropic inversion. Amplitude preserved processing is the prerequisite of inversion. The key to the amplitude preservation data processing is to eliminate the interference brought about by the non-geological factors in order to keep the integrity of the seismic data. Only reliable seismic data can provide true amplitude variation information for the fracture inversion.
From processing flow (Fig. 3), we can see the results are divided into two types: conventional processing flow without pre-stack migration (left part) and processing flow with prestack migration (right part). Fracture inversion will be carried out based on the data from the two flows respectively, in order to test the effect of migration on inversion. Now, we present some key steps in the processing flow:

1) Noise attenuation. Different noise attenuation methods are used considering different noise sources and types. Spherical divergence compensation is applied to improve $\mathrm{S} / \mathrm{N}$.

2) Surface consistent processing. Surface-consistent amplitude compensation, surface-consistent static correction, surface-consistent wavelet shaping and surface-consistent de-convolution are included. Surface consistent amplitude compensation eliminates imbalance of energy resulted from different explosion and receiving conditions. Surface consistent wavelet shaping and surface consistent deconvolution focus on removing inconsistency of the energy in space, instability of waveform and differences between wavelet and phase from different shots, as well as suppressing noise to improve data quality.

3) Forming super gathers. When seismic data from the conventional acquisition is used in inversion, they always lack enough folds, and the azimuth distribution is also limited. Forming super gathers can increase folds and the azimuth range of the azimuth gathers and $\mathrm{S} / \mathrm{N}$ will be improved.

4) Defining azimuth and forming azimuth gathers. The data is divided into groups according to their azimuth and offset range and then the azimuth angle of each gather is defined to form azimuth gathers. 
5) Pre-stack 4D noise attenuation. According to the unpredictability of random noise and the predictability of effective signal in the F-XYZ domain, a prediction operator is applied to the seismic data to suppress noise, and then the data is transformed back into the time-space domain. As a result, noise is suppressed and $\mathrm{S} / \mathrm{N}$ is improved.

6) Multiples elimination. In the Radon domain, the primary wave and multiples are separated based on their different energy positions. Radon Transform is a type of 'subtraction method' when it is used in multiples elimination. First, data are transformed into the Radon domain and the method of velocity removal is used to separate multiples, and then the data are transformed to the time-space domain and multiples are removed from the effective signal.

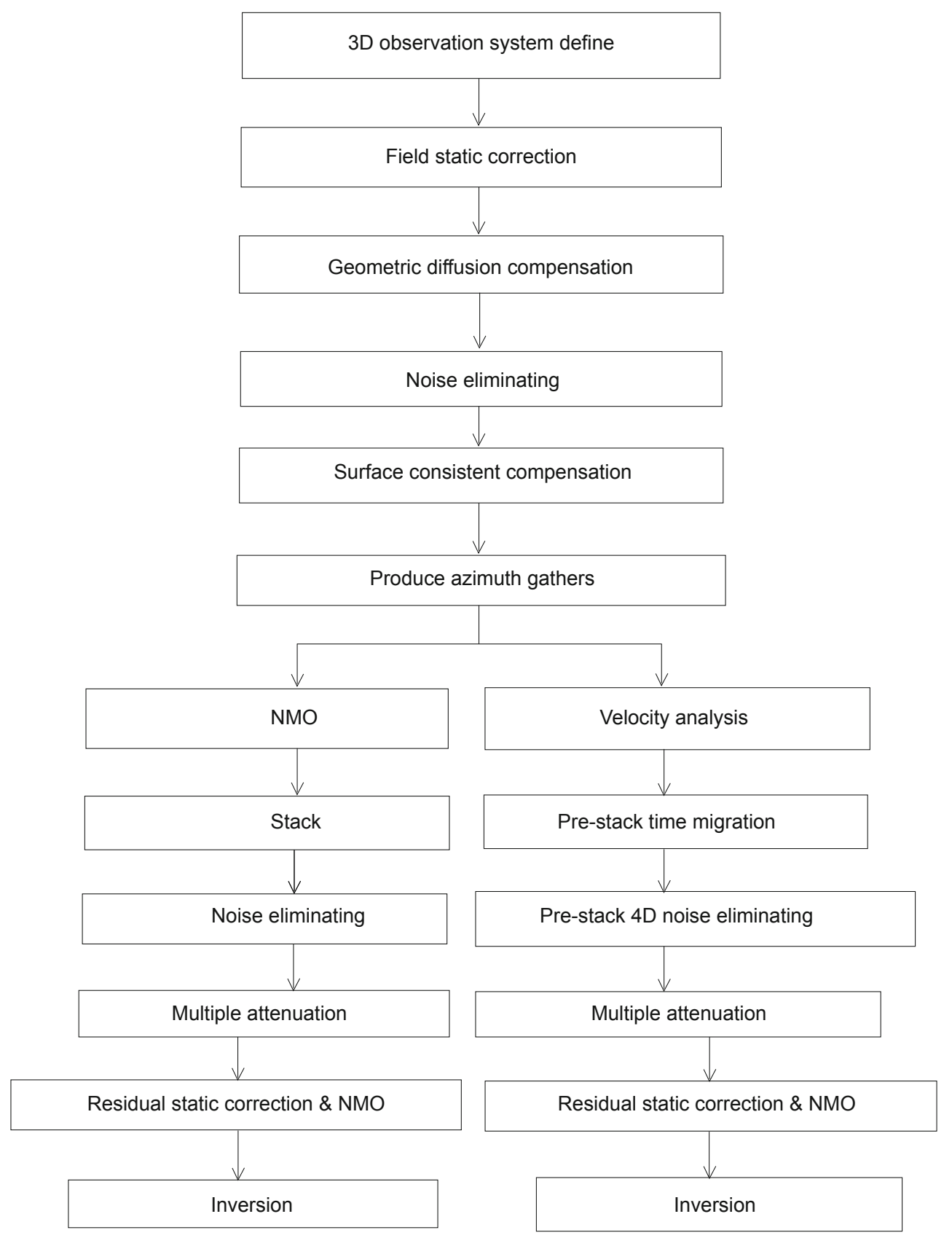

Fig. 3 Pre-stack processing flows of 3D seismic data obtained in the Tazhong carbonate reservoir

\section{Analysis of azimuth gathers before inversion}

3D seismic data in the Tazhong45 area is distributed in the azimuth range of 0-59.3 degrees. The azimuth is divided into four sectors: 0-14.3 degrees, 14.3-29.3 degrees, 29.3-44.3 and 44.3-59.3 degrees. For each sector, the azimuth angle is defined as the middle angles, which are, 7.15, 21.8, 36.8 and 51.8 degrees, so that each azimuth gather in the Tazhong45 area contains four azimuths.

After a series of processing steps, common reflection 
point azimuth gathers are obtained (here, the data processing uses the flows on the right side of Fig. 3). There are four azimuth angles in each reflection binning. If there is no azimuth anisotropy in the medium, the reflection coefficients in different azimuths will be the same. In contrary, if azimuth anisotropy exists, the reflection coefficients in different azimuths will be different. Fig. 4 is an illustration of the azimuth anisotropy.

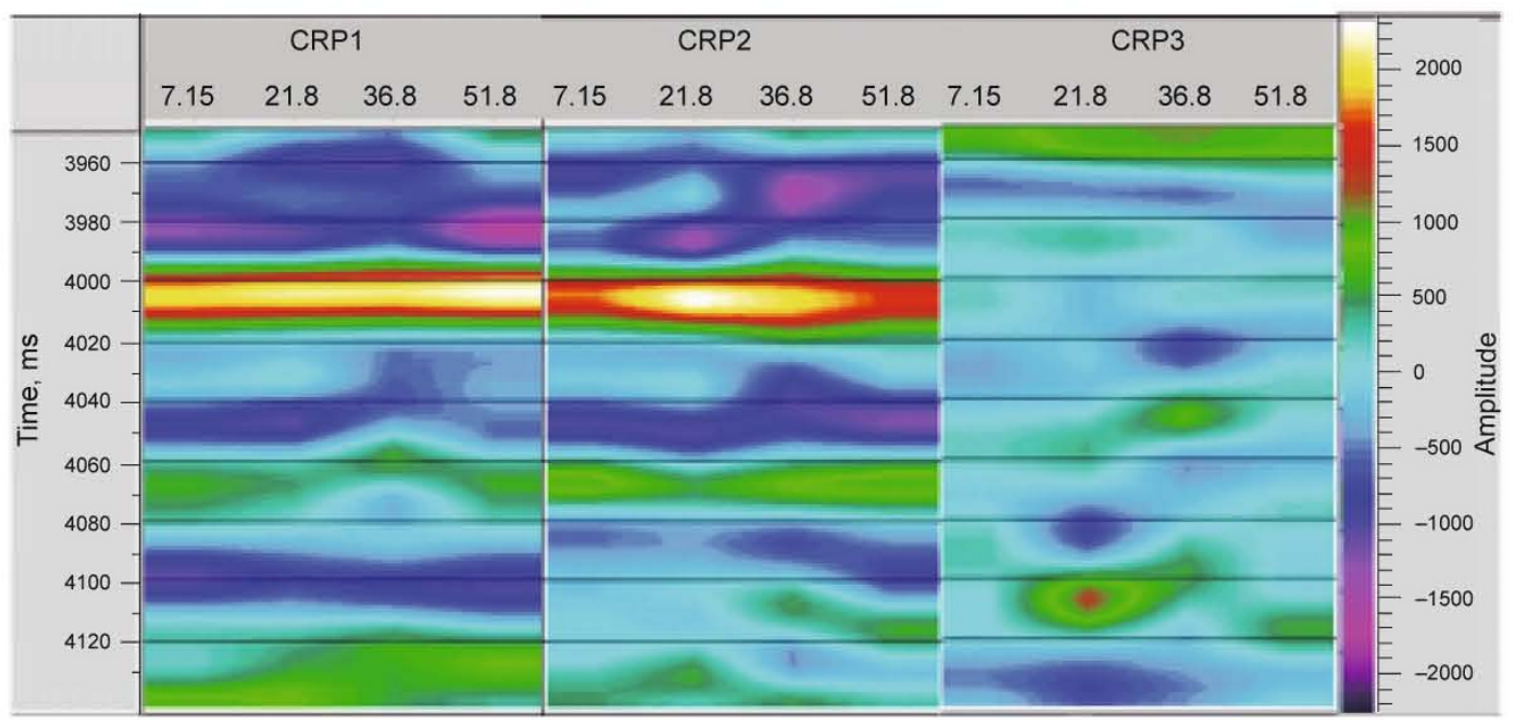

Fig. 4 Azimuth gathers. In CRP1, the amplitude differences among the four azimuths are relatively small, no azimuth anisotropy is displayed. In CRP2, the high amplitudes appear in 21.8 degrees and 36.8 degrees. But in CRP3, no apparent high amplitude is shown because it is located in the range of a fault

\section{The application of the inversion method}

\subsection{Analysis of inversion results}

P-wave anisotropic fracture detection can predict effective fractures which are open or filled with fluid (Lynn et al, 1996). Generally, the effective fractures are highly conductive due to their lower resistance than the surroundings. On the FMI image, dark cosine curves or vertical belts usually show the effective fractures.

Theoretically, the inversion result of fracture detection is influenced by the strike and dip of fractures underground. For the cracks with the same strike and a high dip angle, the larger the linear density (the number of fractures per meter) is, the stronger the seismic wave anisotropy will be. On the contrary, for the cracks with several directions, anisotropy would be relatively weak, which might make the method less effective.

The fracture detection method using limited azimuth seismic data is applied to the case of the Ordovician carbonate reservoir in the TZ45 area. One of the most effective ways to plot fracture data is to combine fracture density and fracture azimuth together using the vector (arrow) plotting technique. The fracture density is represented by the length of an arrow and the azimuth direction is represented by the orientation of the arrow. The longer the arrow bar (vector) the higher the fracture density. The bars are also color-coded by fracture density (warm colors represent high values). The main panel in Fig. 5 shows a horizon map of the vector plot (the integrated display of the fracture density and direction) overlain by a fault map (white lines) from 3D seismic interpretation (coherence cube analysis). The time window for the fracture density extraction is from the top of the Lianglitage II Member to $10 \mathrm{~ms}$ downward from it. The fracture direction is extracted along the top of the Lianglitage II Member.

Fig. 5 shows some fracture characteristics. The area with higher crack density mostly centralizes in the region close to well TZ452. We count the number of effective fractures within the thickness range of the Lianglitage II Member from FMI analysis for 3 wells, and mark the thickness of the strata corresponding to the Lianglitage II Member for each of the wells (e.g. $4 \mathrm{n} / 55 \mathrm{~m}$ means 4 effective fractures within the thickness of $55 \mathrm{~m}$ in the Lianglitage II Member in well TZ86). Moreover, rose diagrams are employed based on the statistics from FMI. The radial length represents the number of cracks and the axis angle shows the strikes of fractures. As is shown in Fig. 6, for the fractures with the uniform (same) strike, the fracture density is relatively high (wells ZG26 and ZG16); while for the fractures with different strikes, the inverted fracture density is relatively low (well TZ86). Anisotropy is the comprehensive effect of multi-groups of fractures underground, which makes the anisotropic inversion method less effective if the fracture strikes are different. The fracture density increases from southeast to northwest.

The fracture linear density and crack filling characteristics of the grain limestone member (this member is consistent with the Lianglitage II Member) are described based on core data and FMI logs now available. In the plane, the fault orientations have two dominant ones, where those are Northwest-Southeast and Northeast-Southwest. Fig. 6 gives detailed statistic results. The linear fracture density increases 


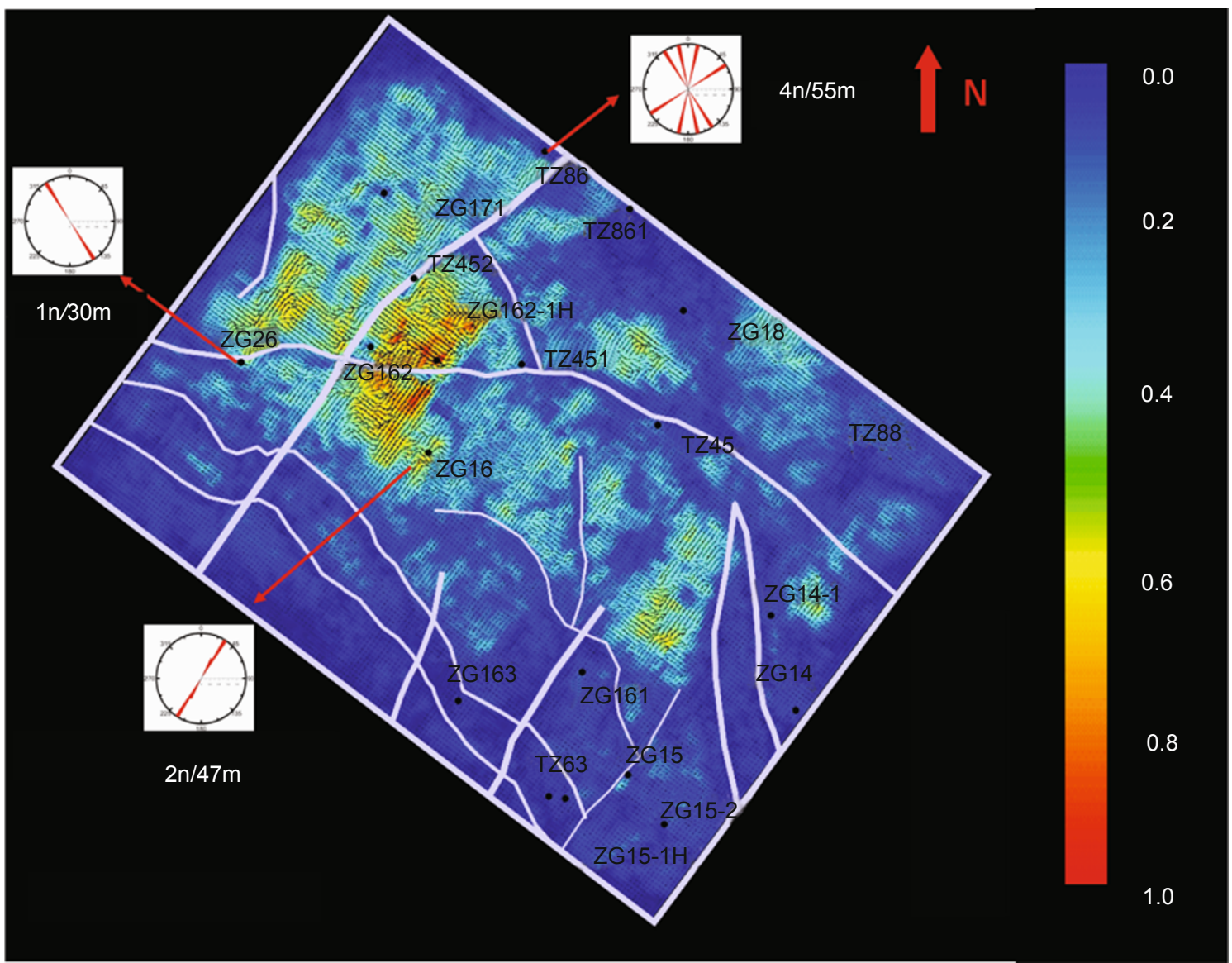

Fig. 5 Fracture density and direction inverted in the TZ45 area. The area with higher crack density mostly centers on the region close to well TZ452. Fracture density increases from southeast to northwest

from well TZ88 to well ZG17 (from FMI data, the fracture linear density of well TZ88 equals to 0.06 , well TZ86 shows 0.09 and well ZG17 shows 0.17 ). The fractures which are of high conductivity identified by the FMI well logging data of well TZ88 account for $43 \%$ in the whole number of fractures in this well, while fractures of high conductivity of well TZ86 account for $78 \%$, and those of well ZG17 account for $86 \%$. Based on the core data, well TZ49 and well TZ63 are all fully filled, the filling degree decreases to well TZ45 and well TZ451, half filled fractures account for $56.3 \%$ and $62.5 \%$ in the two wells above respectively. For well TZ86, the filling degree is the lowest, the half filled and unfilled fracture account for $63 \%$. Therefore, from FMI logging data and core data we draw the conclusion that the filling degree of the fractures decreases from southeast to northwest and from southwest to northeast, which indicates effective fractures increase from southeast to northwest. Therefore, the inversion result is in good agreement with the geological background in this area.

To further illustrate the effectiveness of the inversion method, the azimuth gathers across well ZG16, the inverted results (fracture density and direction) across well ZG16 and the FMI data from this well are compared.

The prestack azimuth gathers across well ZG16 (inline 1206, crossline 1004) are extracted. The offsets extracted are 2,575, 2,585 and 2,595 $\mathrm{m}$ respectively, and the azimuth angles corresponding to each offset are 7.15, 21.8, 36.8, and 51.8 degrees. As it is shown in Fig. 7, the amplitudes in 36.8 degrees for each of the azimuth gather are higher, thus there is a certain amount of anisotropy in these azimuth gathers.

In Fig. 8, the inversion results (fracture density and direction) across well ZG16 and the FMI data of well ZG16 are compared. The fracture density section is on the left side of Fig. 8(a), and the right one is part of the FMI image between the horizons on top of the Lianglitage II Member and $10 \mathrm{~ms}$ downward from it, the FMI image shows effective fracture (dark cosine lines, which represent the highly conductive fractures). The figure on the left side of Fig. 8(b) is the amplified result of Fig. 5 near well ZG16, and the right figure is the statistical FMI rose diagram.

From the fracture density section (left of Fig. 8(a)), we can see that the fracture density is quite high between the top of the Lianglitage II Member and the horizon $10 \mathrm{~ms}$ downward from it. The FMI image (right of Fig. 8(a)) and its rose diagram show that the fracture direction is 35 degrees north by east. The amplified result near well ZG16 (left of Fig. 8(b)) show that the fracture direction is in good agreement with the FMI data. From the azimuth gathers across well ZG16, the inverted results (fracture density and direction) across well ZG16 and the FMI data from this well, we can conclude that the inversion results are consistent with the well data, which suggests that the inversion method is reliable and applicable. 


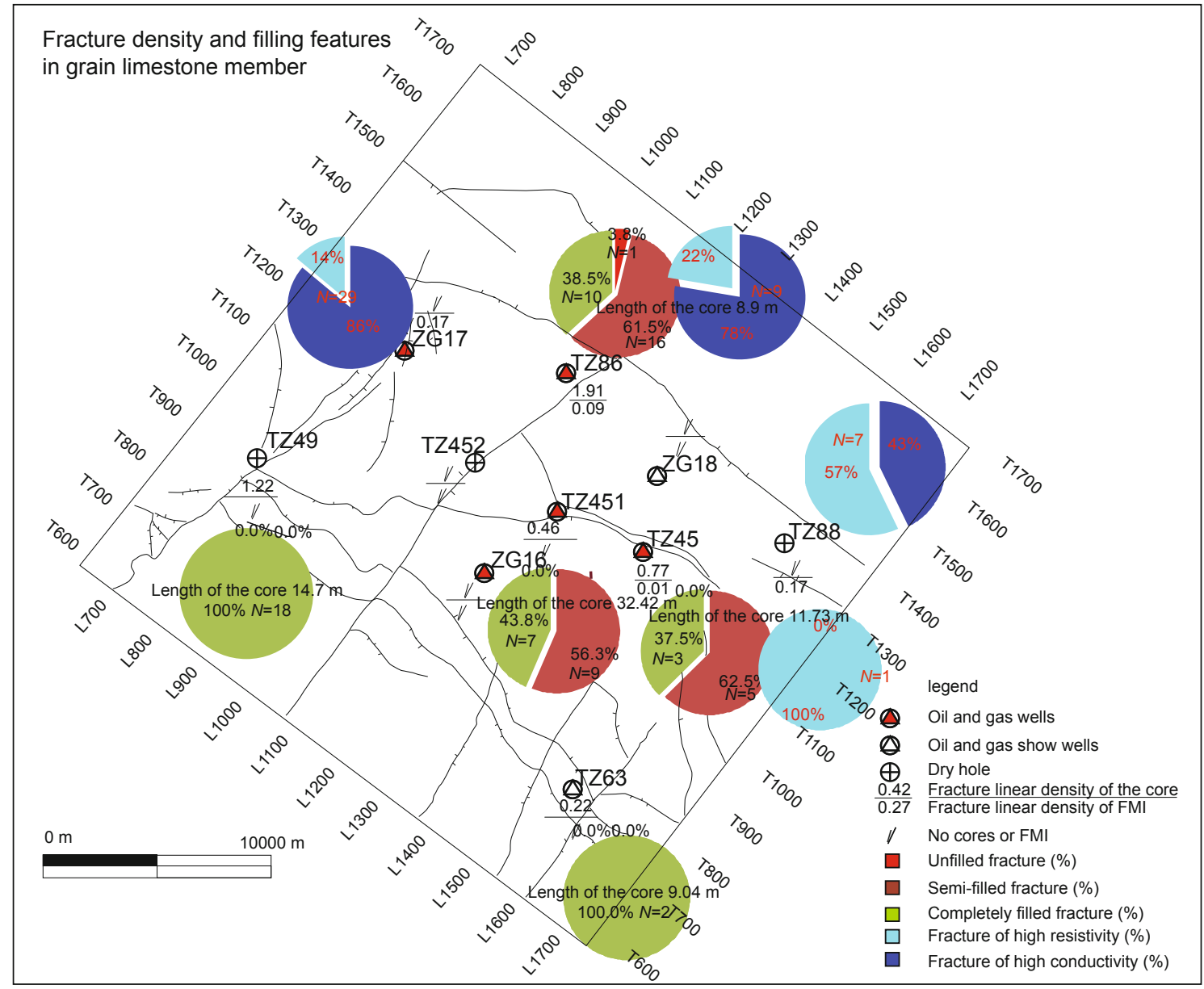

Fig. 6 Linear fracture density and filling features in grain limestone member

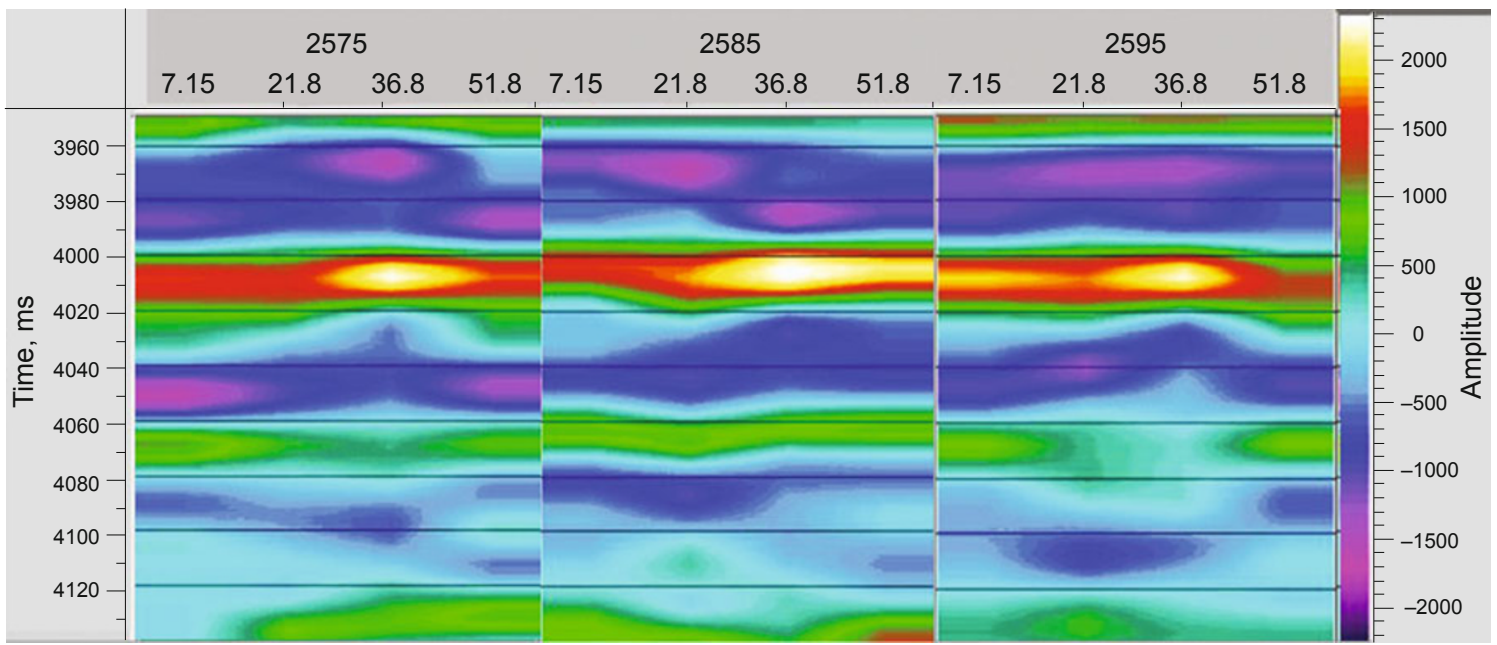

Fig. 7 Azimuth gathers across well ZG16

\subsection{Effects of migration on inversion}

The fracture prediction algorithm is applied to two sets of data (data that went through migration and data that did not), and the results include two parameters: fracture density and fracture direction (Figs. 9 and 10).
Figs. 9 and 10 show the inversion results (fracture density and direction) of top of the Lianglitage II Member. The left part of each figure represents the inverted fracture density, while the right part represents the fracture direction. It can be found that inversion results using the ordinary data which has 

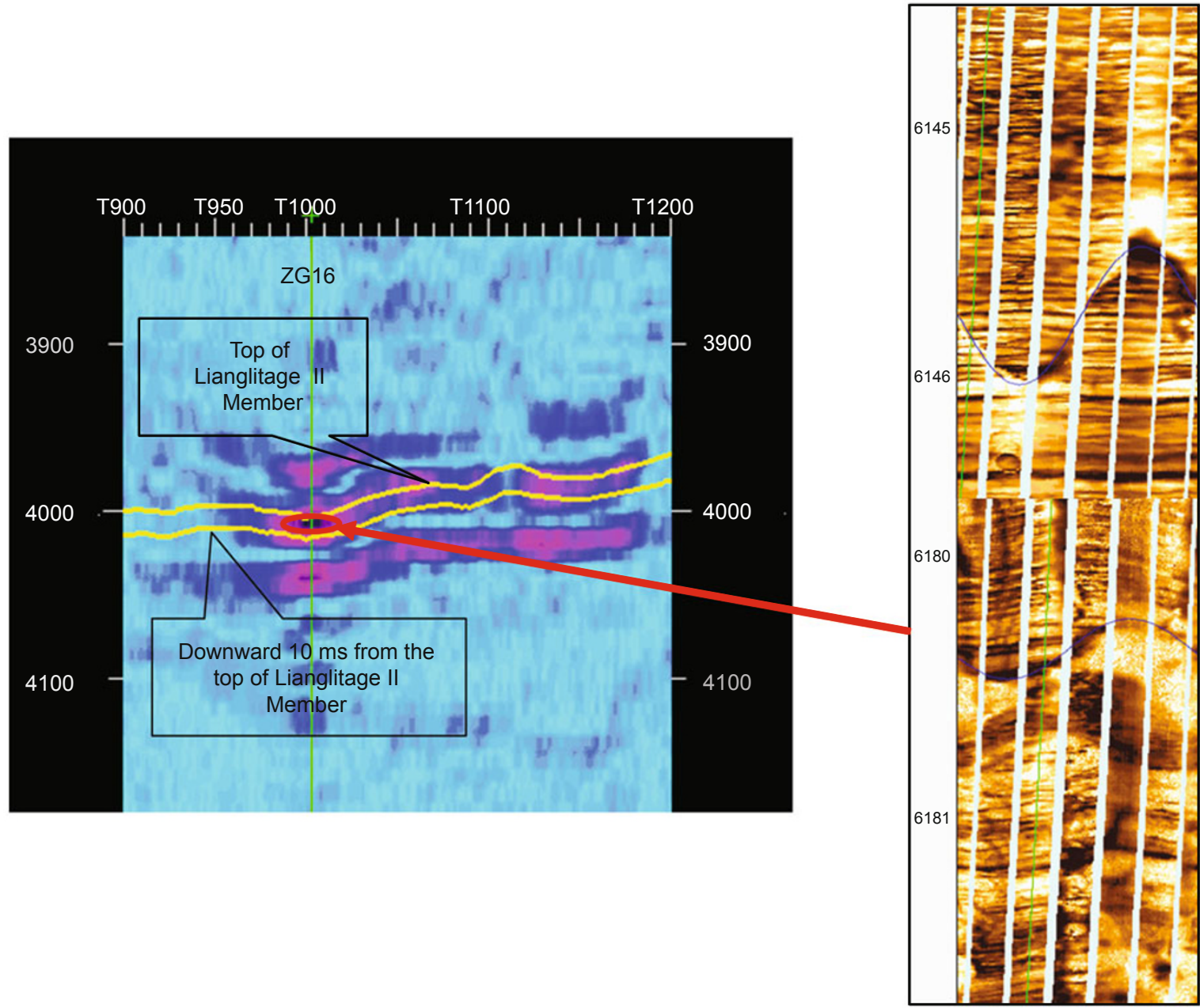

(a) Comparison of the inverted fracture density section and FMI data

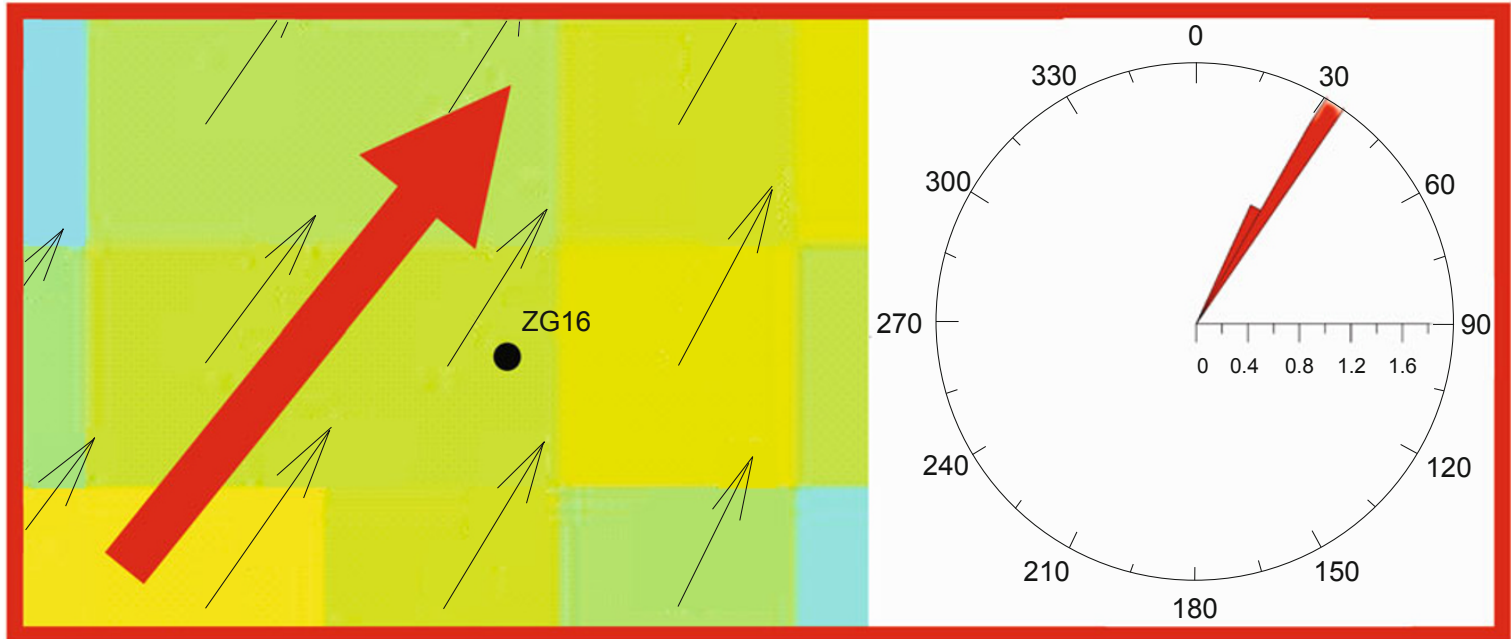

(b) The amplified inverted result near well ZG16 and the rose diagram obtained from FMI

Fig. 8 Comparison between the inverted result and FMI

not gone through migration can not reflect the information of fractures, while the data after pre-stack migration can. This is because migration can provide the true amplitude variation which is significant to inversion. Because of the complexity of the geological conditions and seismic data in the Tazhong area, migration should be included in the processing flow. 

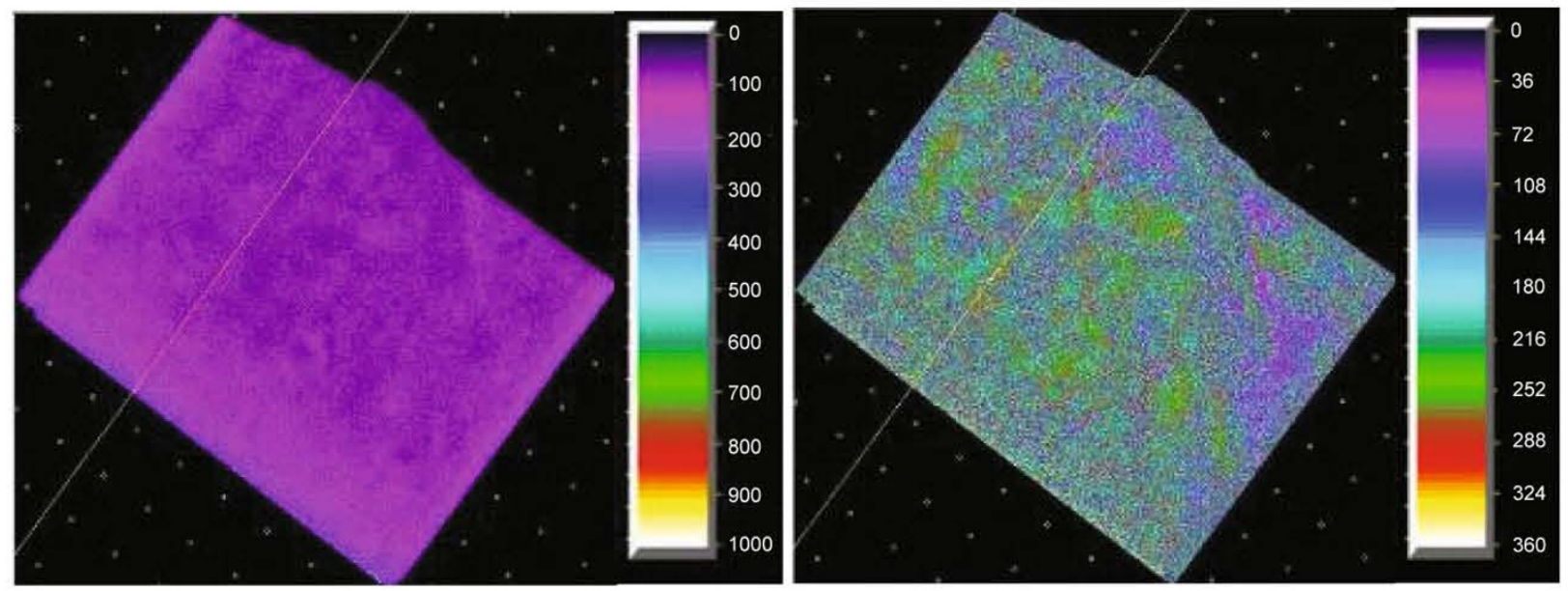

Fig. 9 Fracture density and direction from data resulting from ordinary processing (without migration) in the TZ45 area

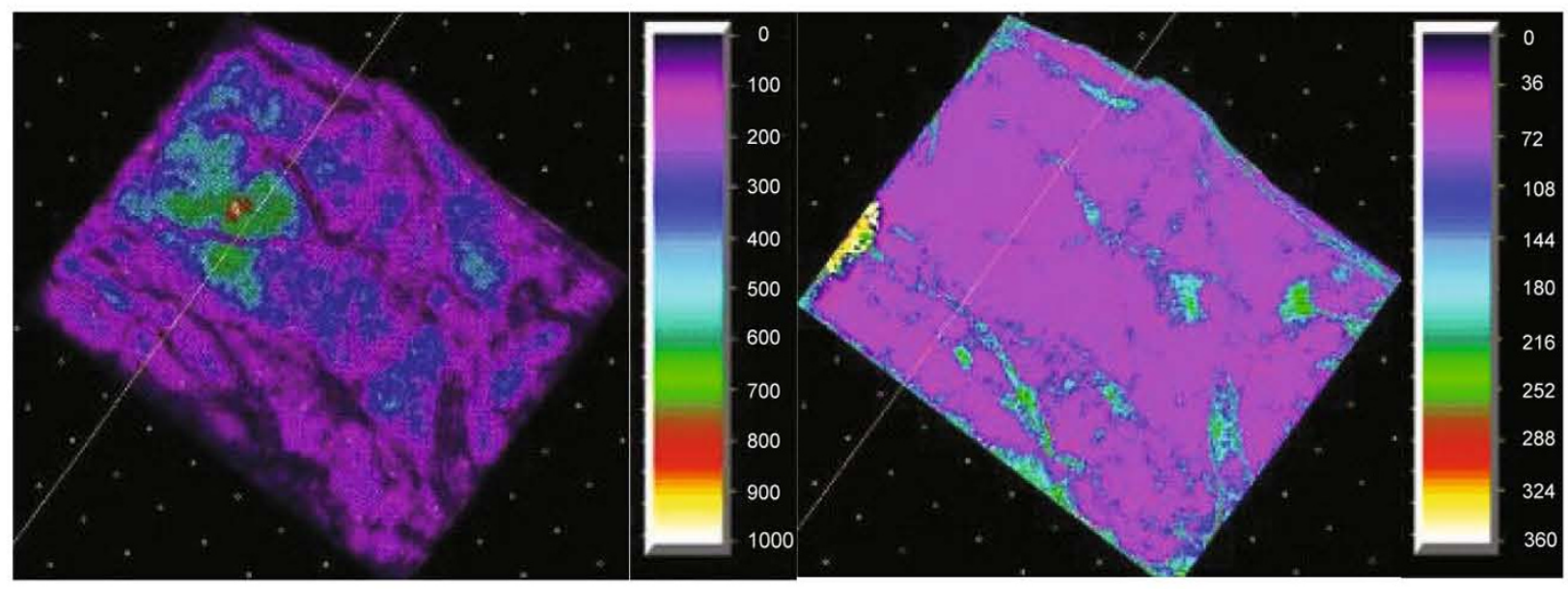

Fig. 10 Fracture density and direction from data resulting from pre-stack migration in the TZ45 area

\section{Conclusions}

1) The conventional fracture detection method requires the use of wide azimuth data, because the existence of noise will make the solution unstable if the data azimuth is narrow or limited. However, the wide azimuth field data is not always available due to the high cost of acquisition. To solve the problem, a noise attenuation algorithm is adopted in fracture prediction using data with limited azimuth distribution, ensuring the stability of the solution. The feasibility has been tested by numerical examples. Compared with the conventional method, this inversion technique could greatly reduce the cost of acquisition.

2) The inversion method assumes the reflection amplitude of the seismic wave to be the reflection coefficient, and the wavelet is not extracted from the seismic data, thus the inverted fracture density will be influenced by the sidelobe of the wavelet and the effect of wavelet stacking. Therefore, in the inverted fracture density section, the position with higher fracture density does not necessarily imply that it is the anisotropic interface and anisotropy is strong here, because it might be affected by the wavelets. Here, we only consider the main anisotropic interface, that is, the relatively apparent horizontal interface in the prestack gathers. Meanwhile, another precondition when the reflection amplitude is assumed to be the reflection coefficient is that the wavelets in each azimuth are the same. Further research requires error testing for this type of inversion.

3) Data processing flows which can preserve the amplitude integrity have been implemented in the study area, multiples elimination and $\mathrm{S} / \mathrm{N}$ enhancement have been carried out. Migration is important to inversion.

4) The fracture detection method is applied to the data with limited azimuth distribution in the TZ45 area, Tarim Basin. Compared with the statistic results of fracture features from FMI and core, the inversion results are reasonable, suggesting the feasibility of the proposed fracture inversion method.

\section{Acknowledgments}

The authors would like to thank the Laboratory for Integration of Geology and Geophysics (LIGG) at China University of Petroleum for the permission to publish this work and we appreciate Xie Chunhui, Bai Haijun and Zhao Haitao for their discussion in the research. 


\section{References}

Al-Marzoug A, Neves F A, Kim J J and Nebrija E. P-wave anisotropy from azimuthal $\mathrm{AVO}$ and velocity estimates using $3 \mathrm{D}$ seismic data from Saudi Arabia. Geophysics. 2006. 71(2): E7-E11

Crampin S, McGonigle R and Bamford D. Estimating crack parameters from observations of P-wave velocity anisotropy. Geophysics. 1980 45(3): $345-360$

Gray D and Head K. Fracture detection in Manderson Field: A 3-D AVAZ case history. The Leading Edge. 2000. 19(11): 1214-1221

Grechka V, Tsvankin I and Cohen J K. Generalized Dix equation and analytic treatment of normal-moveout velocity for anisotropic media. Geophysical Prospecting. 1999. 47(2): 117-148

Luo M and Evan B J. An amplitude-based multiazimuth approach to mapping fractures using P-wave 3D seismic data. Geophysics. 2004 69(3): 690-698

Lynn H B, Simon K M and Bates C R. Correlation between P-wave AVOA and S-wave traveltime anisotropy in a naturally fractured gas reservoir. The Leading Edge. 1996. 15(8): 931-935

Mallick S, Craft K L, Meister L J, et al. Determination of the principal directions of azimuthal anisotropy from P-wave seismic data. Geophysics. 1998. 63: 692-706

Neidell N S and Cook E E. Seismic method for identifying low velocity subsurface zones. US Patent. 1986. 4571710

Ruger A. Reflection coefficient and azimuthal AVO analysis in anisotropic media. Ph.D Thesis. 1996. Colorado School of Mines, Leadville, Colorado

Ruger A. Variation of P-wave reflectivity with offset and azimuth in anisotropic media. Geophysics. 1998. 63(3): 935-947

Schoenberg M A, Dean S and Sayers C M. Azimuth dependent turning of seismic waves reflected from fractured reservoirs. Geophysics. 1999. 64(4): 1160-1171

Shen F, Sierra J, Burns D R and Toksöz M N. Azimuthal offsetdependent attributes applied to fracture detection in a carbonate reservoir. Geophysics. 2002. 67(2): 355-364

Sun Z D and Wang Y Y. P-wave fracture prediction algorithm using prestack data with limited azimuthal distribution. 2008. Research Report Vol. 1 of Laboratory for Integration of Geology and Geophysics, China University of Petroleum. 13-23

Sun Z D, Jia C, Zhou X, et al. Carbonate research in China-technologies meeting tough challenges. 2011a. EAGE, 73rd annual meeting, Expanded Abstract

Sun Z D, Xiao X and Wang Z M. P-wave fracture prediction algorithm using prestack data with limited azimuthal distribution. 2011b. EAGE, 73rd annual meeting, Expanded Abstract

Tsvankin I D. Seismic Signatures and Analysis of Reflection Data in Anisotropic Media. Pergamon Press. 2001

Wang D Y, Lu C P, Dong W J, et al. Fracture analysis for carbonate reservoirs using 3D seismic P-wave data: Middle East case study. Paper SPE 101596 presented at Abu Dhabi International Petroleum Exhibition and Conference, 5-8 November 2006, Abu Dhabi, UAE

Zelewski G, Lu C P, Tsenn M, et al. P-wave seismic azimuthal anisotropy for detection and prediction of fractures in a Middle Eastern carbonate reservoir. 2009. International Petroleum Technology Conference, 13903

(Edited by Sun Yanhua) 